\title{
CLINICAL RESEARCH ARTICLE High incidence of stroke and mortality in pediatric critical care patients with COVID-19 in Peru
}

\author{
Alvaro Coronado Munoz ${ }^{1}$, Jaime Tasayco ${ }^{2}$, Willy Morales ${ }^{2}$, Luis Moreno ${ }^{2}$, David Zorrilla ${ }^{2}$, Angie Stapleton ${ }^{3}$, Patricia Pajuelo ${ }^{3}$, \\ Giuliana Reyes $^{3}$, Matilde Estupiñan ${ }^{3}$, Ricardo Seminario ${ }^{3}$, Manuel Ortiz $^{3}$ and Jesús Domínguez ${ }^{2}$
}

INTRODUCTION: Pediatric critical care patients with COVID-19 treated in Peru have higher mortality than those previously reported from other countries. Pediatric providers have reported a high number of patients without comorbidities presenting with hemorrhagic strokes associated with COVID-19. We present a study analyzing the factors associated with mortality in this setting. METHODS: Prospective case-control study that included patients $<17$ years old admitted to a pediatric critical care unit with a positive test confirming COVID-19. The primary outcome was mortality. Fisher's exact test and the Mann-Whitney $U$ test were used for the analysis.

RESULTS: Forty-seven patients were admitted to critical care. The mortality of our study is $21.3 \%$. The mortality of patients with neurological presentation was $45.5 \%$, which was significantly higher than the mortality of acute COVID-19 (26.7\%) and MIS-C (4.8\%), $p$ 0.18. Other risk factors for mortality in our cohort were strokes and comorbidities. Only one patient presenting with hemorrhagic stroke had an undiagnosed comorbidity.

CONCLUSION: Cerebrovascular events associated with COVID-19 in pediatric patients, including infants, must be recognized as one of the more severe presentations of this infection in pediatric patients.

Pediatric Research (2022) 91:1730-1734; https://doi.org/10.1038/s41390-021-01547-x

\section{IMPACT:}

- Pediatric patients with COVID-19 can present with hemorrhagic and ischemic strokes on presentation.

- Neurological presentation in pediatric patients with COVID-19 has high mortality.

- Mortality of pediatric patients with COVID-19 is associated with comorbidities.

- Pediatric presentation and outcomes of COVID-19 in different regions can be novel to previously described.

\section{INTRODUCTION}

The mortality of pediatric patients with COVID-19 acute respiratory failure has been mainly associated with comorbidities. ${ }^{1-3}$ Epidemiological studies from different countries report mortalities of $0.1-0.3 \%$ for all patients hospitalized with COVID-19. The novel presentation of multisystemic inflammatory syndrome in children (MIS-C) alerted the medical community about the pathogenesis of COVID-19 in pediatric patients. ${ }^{4-8}$ The broad definition proposed by the CDC includes inflammatory processes in different systems, such as cardiac, renal, neurological, respiratory, and gastrointestinal systems. The severity of MIS-C has been mostly associated with cardiac complications ranging from severe heart failure and coronary aneurisms. ${ }^{4,6,8-11}$ However, the mortality in epidemiological studies of this syndrome is also low, reported as high as $2 \%$, with most complications associated with cardiac failure and shock. The mortality and morbidity of COVID-19 in pediatric critical care patients has been described in different studies. ${ }^{12-14}$ The mortality in those studies was also low, ranging from 2 to $6 \%$ for patients admitted to critical care units. One of the largest studies from pediatric critical care units (PICUs) reported a mortality of $5.7 \%$ from 530 patients from the United States and Canada databases. ${ }^{15}$
Since the emergency state was declared for the COVID-19 pandemic, one of the measures adopted by the government in Peru was to create a dedicated COVID-19 hospital. Within that hospital, a six-bed pediatric critical care unit was implemented. When the capacity of that unit was reached with acute COVID-19 cases, other hospitals were able to admit patients with MIS-C. The mortality in pediatric critical care patients in Peru in the dedicated COVID-19 unit is $>20 \%$, significantly higher than that reported in studies with similar populations. ${ }^{15}$ In the past 3 months, different pediatric critical care groups and pediatric emergency groups in Peru alerted an increasing number of pediatric patients presenting to emergency rooms with neurological symptoms and COVID-19. This acute presentation has not been described previously in other settings. We present the cases admitted to the COVID-19 PICU and the data of one additional center that hospitalized MIS-C patients. The purpose is to analyze the causes of mortality for pediatric critical care patients with COVID-19 in Peru.

\section{METHODS}

We performed a prospective case-control study for patients hospitalized from 26 March to 15 August 2020 in two centers in

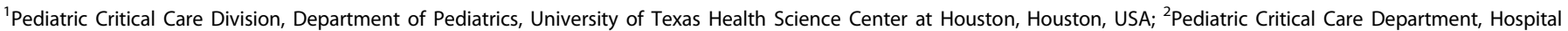
Emergencias Villa el Salvador, Lima, Peru and ${ }^{3}$ Pediatric Department, Hospital Edgardo Rebagliati Martins, Lima, Peru

Correspondence: Alvaro Coronado Munoz (Alvaro.j.coronadomunoz@uth.tmc.edu) 
Lima, Peru. Patients were selected from two hospitals. The first hospital is a COVID-19 center, reporting patients from the pediatric critical care unit. The second hospital included patients admitted to the PICU with MIS-C diagnosis. This study was reviewed by the IRB office at each center: "Comité de Ética en Investigación para COVID-19, ESSALUD" and "Coordinadora de la Unidad de Docencia e Investigación, HEVES". Both IRB committees deemed the study exempted due to the COVID-19 emergency and authorized the completion of the study.

\section{Population}

We included patients $0-17$ years old with positive testing for COVID-19 (PCR, IgM, or IgG) with SARS-CoV-2 or MIS-C admitted to the pediatric critical care unit. We excluded patients with a positive test for COVID-19 but that the admission diagnosis was nonCOVID-19 related (e.g., trauma). The definition of MIS-C followed the criteria defined by the United States CDC. ${ }^{5}$ We divided our patients into three groups by clinical presentation: acute COVID-19, MIS-C, and neurological presentation. Patients with acute COVID-19 were considered patients with respiratory failure requiring mechanical ventilator support. Patients with MIS-C met the United States CDC definition. Patients with neurological presentation were patients with neurological symptoms at admission.

\section{Outcomes and variables}

The primary outcome was mortality during hospitalization. The variables included were demographics (age and sex) and referring center. For therapies, we collected information on treatments considered to be directed COVID-19 treatment in Peru: ivermectin and hydroxychloroquine. We also included the use of steroids, vasoactive medications, and intravenous immunoglobulins. For steroids, we included dexamethasone and methylprednisolone, low dose, and pulse steroid dose. Vasoactive medications included dopamine, dobutamine, epinephrine, and norepinephrine. For anticoagulation therapies, we included antiplatelet medication (aspirin), heparin, and enoxaparin. We included severe respiratory distress syndrome utilizing the Berlin definition of a $\mathrm{PaO}_{2} / \mathrm{FiO}_{2}$ less than 100 (ref. $\left.^{16}\right)$. Laboratory values were collected for all the patients, including in the analysis the worst value (peak value) of the first $96 \mathrm{~h}$ of admission. Brain imaging results were obtained for all the patients with neurological complications and a description of the patients presenting with cerebrovascular accident (CVA), ischemic stroke, or hemorrhagic stroke. Echocardiograms data were also collected. Treatments used in all the patients were also collected.

Statistical analysis

Statistical analysis was performed with frequentist analysis. Continuous variables were analyzed with the Mann-Whitney $U$ test, and categorical variables were analyzed with Fisher's exact test. Statistical analysis was performed with the IBM SPSS 26.0 software package.

\section{RESULTS}

During the study period, a total of 47 patients were admitted; 39 patients were from the COVID-19 unit and 8 were from the second institution. A total of 15 (31.9\%) patients were admitted with SARSCoV-2, 21 (44.7\%) were admitted with MIS-C, and 11 (23.4\%) patients presented neurological complications. Most patients included were male patients, 30 (63.8\%). The age ranged from 1 month to 16 years. Only four patients with neurological presentation had severe or moderate acute lung injury concomitantly. Only five with neurological presentation met MIS-C criteria. Comorbidities were present in 15 (31.9\%) of the patients, with most within the Acute COVID-19 group $(12(80 \%), p<0.01)$. The three groups also differed in steroid use: acute COVID-19 11 (73.3\%), MIS-C 8 (38.1\%), and neurological 9 $(81.8 \%), p$ 0.03. A detailed description of the patients included is presented in Table 1.
Table 1. Characteristics of critical care patients with SARS-CoV2 infection.

\begin{tabular}{|c|c|c|c|c|}
\hline & $\begin{array}{l}\text { Acute } \\
n=15\end{array}$ & $\begin{array}{l}\text { MIS-C } \\
n=21\end{array}$ & $\begin{array}{l}\text { Neurological } \\
n=11\end{array}$ & $p$ value \\
\hline Age, months & $12(6 ; 144)$ & $84(32.5 ; 120)$ & $60(12 ; 156)$ & 0.51 \\
\hline Sex, male & 7 (46.7\%) & $15(71.4 \%)$ & $8(72.7 \%)$ & 0.25 \\
\hline Admission, referred & $11(73.3 \%)$ & $13(61.9 \%)$ & $7(63.6 \%)$ & 0.79 \\
\hline Out-of-city transfer & $3(20 \%)$ & 2 (9.5\%) & $2(18.2 \%)$ & 0.66 \\
\hline Comorbidities & $12(80 \%)$ & $1(4.8 \%)$ & $2(18.2 \%)$ & $<0.01$ \\
\hline \multicolumn{5}{|l|}{ Kawasaki } \\
\hline Complete & - & 7 (33.3\%) & - & - \\
\hline Atypical & & $6(28.6 \%)$ & $1(9.1 \%)$ & \\
\hline $\mathrm{PaO}_{2} / \mathrm{FiO}_{2}<100$ & 7 (46.7\%) & - & $1(9.1 \%)$ & 0.17 \\
\hline LOS PICU, days & $6(3 ; 15)$ & $6(4.5 ; 10)$ & $9(7 ; 12)$ & 0.35 \\
\hline LOMV, days, $n=15$ & $7(3 ; 15)$ & $7(4.25 ; 10.5)$ & $6.5(3.5 ; 8.5)$ & 0.47 \\
\hline Mortality & $4(26.7 \%)$ & $1(4.8 \%)$ & $5(45.5 \%)$ & 0.02 \\
\hline Steroids & $11(73.3 \%)$ & $8(38.1 \%)$ & $9(81.8 \%)$ & 0.03 \\
\hline Vasoactive med. & $6(40 \%)$ & $6(28.6 \%)$ & 7 (63.6\%) & 0.17 \\
\hline $\begin{array}{l}\text { COVID directed } \\
\text { therapy }\end{array}$ & $4(26.7 \%)$ & $6(28.6 \%)$ & $4(36.4 \%)$ & 0.84 \\
\hline \multicolumn{5}{|l|}{ Anticoagulation } \\
\hline ASA & - & 9 (42.9\%) & - & $<0.01$ \\
\hline$A S A+L M W H$ & - & $6(28.6 \%)$ & - & \\
\hline LMWH & $2(13.3 \%)$ & $2(9.5 \%)$ & $4(36.4 \%)$ & \\
\hline IVIG & - & 15 (71.4\%) & 1 (9.1\%) & - \\
\hline
\end{tabular}

Continuous variables expressed in median and interquartile ranges in parenthesis. Analysis: continuous variables Mann-Whitney $U$ test; categorical Fisher's exact test. LOS length of stay, LOMV length of mechanical ventilation, $L M W H$ low-molecular weight heparin.

The mortality of our study was $21.3 \%$, and 10 patients died during the study period. The mortality of patients with neurological presentation was $45.5 \%$, which was significantly higher than the mortality of acute COVID-19 (26.7\%) and MIS-C (4.8\%), $p$ 0.18. Other risk factors of mortality vs survival in our cohort were comorbidities 7 (70\%) vs 8 (21.6\%), p 0.007, strokes 4 (40\%) vs $2(5.4 \%), p 0.014$ and higher white blood cell count, median, and interquartile ranges for mortality $21(14 ; 31)$ vs $13(9 ; 16)$ for survival, $p 0.01$. Therapies and mode of admission were not associated with mortality. Severe acute lung injury, defined by a $\mathrm{PaO}_{2} / \mathrm{FiO}_{2}$ value of less than 100 at $24 \mathrm{~h}$ of admission, was not associated with mortality. The CRP level was not associated with higher mortality. We present these variables analyzed for mortality in Table 2.

Cerebrovascular events occurred in six patients (12.8\%) in our cohort, and four of them died. Most patients had hemorrhagic stroke, and only one patient had an ischemic stroke. One patient with hemorrhagic stroke and one patient with ischemic stroke survived but had significant sequelae. The presentation of these patients varied from seizures to vomiting. There was no previous use of anticoagulation therapies or trauma in any of those patients. One patient with hemorrhagic stroke had a comorbidity, and a diagnosis of leukemia was made 2 days after admission. A detailed description of neurological cases can be seen in the Supplementary Material. Brain tomography images on admission are shown in Fig. 1.

Comorbidities in our study were associated with mortality. For the group presenting with acute COVID-19, all mortalities were associated with comorbidities: one patient had acute lymphocytic leukemia, another patient had cerebral palsy and hypoxic ischemic encephalopathy, the third patient had trisomy 21 and an unrepaired ventricular septal defect, and finally, the last patient 
Table 2. Associations with mortality.

\begin{tabular}{|c|c|c|c|}
\hline & Survived & Mortality & \\
\hline Age & $60(9 ; 126)$ & $37(2.75 ; 138)$ & 0.63 \\
\hline Comorbidities & $8(21.6 \%)$ & 7 (70\%) & 0.007 \\
\hline Vasoactive med. & $14(37.8 \%)$ & $5(50 \%)$ & 0.72 \\
\hline COVID-19 directed therapy & $9(24.3 \%)$ & $5(50 \%)$ & 0.14 \\
\hline Steroids & $21(56.8 \%)$ & $7(70 \%)$ & 0.72 \\
\hline Stroke & $2(5.4 \%)$ & $4(40 \%)$ & 0.014 \\
\hline Admission, referral & $24(64.9 \%)$ & $7(70 \%)$ & 1 \\
\hline Out-of-city transfer & $5(13.5 \%)$ & $2(20 \%)$ & 0.63 \\
\hline $\mathrm{PaO}_{2} / \mathrm{FiO}_{2}<100$ & $4(22.2 \%)$ & $4(50 \%)$ & 0.2 \\
\hline$W B C \times 10^{3}$ & $13(9 ; 16)$ & $21(14 ; 31)$ & 0.01 \\
\hline CRP & $33.7(14.1 ; 226)$ & $32.6(5.1 ; 274)$ & 0.98 \\
\hline Platelets & $234(142 ; 509)$ & $147(54 ; 369)$ & 0.12 \\
\hline
\end{tabular}

Continuous variables expressed in median and interquartile ranges in parenthesis. Analysis: continuous variables Mann-Whitney $U$ test; categorical Fisher's exact test. med. medications, WBC white blood cell count, CRP C-reactive protein $(\mathrm{mg} / \mathrm{L})$.

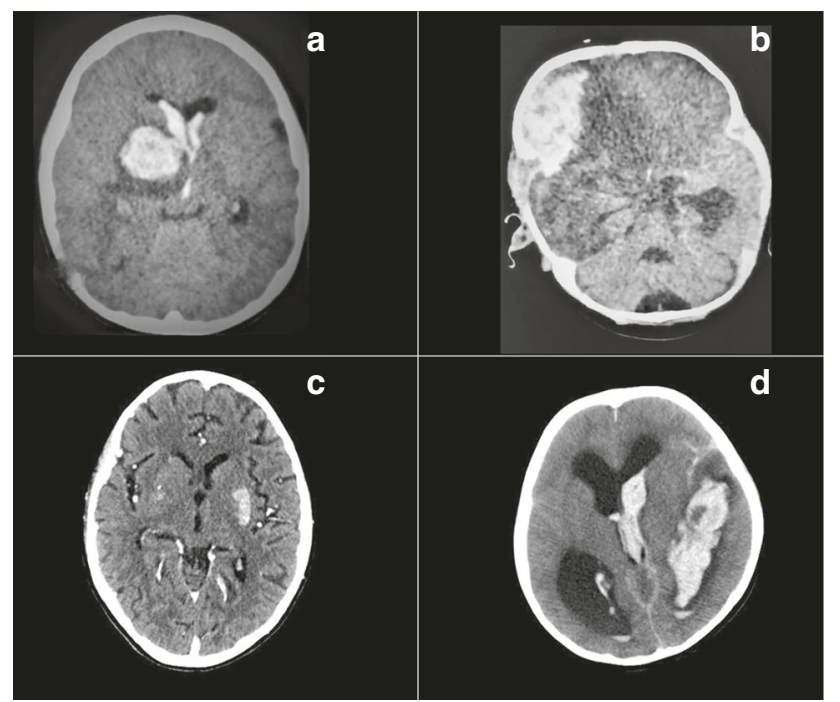

Fig. 1 Brain images of 4 patients with Hemorrhagic strokes presentation. a basal ganglia hemorrhage, anterior horns of lateral ventricles; b right frontal hemorrhage, transtentorial herniation; c hypodensity in left basal ganglia, ischemic stroke, diffuse subcortical atrophy; d ventricular hemorrhage and hydrocephalus.

had undiagnosed Epstein anomaly. The patient with MIS-C who died during the period of study had a history of nephrotic syndrome and dialysis-dependent kidney failure. For the patients in the neurological group, one of them had pulmonary tuberculosis and developed tuberculosis meningitis, initially thought to be COVID-19 encephalitis, and the other patient was the undiagnosed patient with leukemia who presented with a hemorrhagic stroke. Differences between the three groups associated with morbidity and management can be found in Table 1; laboratory results by admission diagnosis can be found in Fig. 2 .

\section{DISCUSSION}

Our study presents risk factors for mortality in pediatric critical care patients with COVID-19 in Peru. The mortality rate in our cohort was $21 \%$. Mortality was higher in patients with comorbidities, strokes, and higher white blood cell count. One significant finding is the association of mortality of patients with neurological presentation. The presentation on admission of hemorrhagic strokes in pediatric patients with COVID-19 has not been described in other populations. Recognizing this presentation is extremely important for the medical community working with pediatric patients to rapidly diagnose and manage strokes.

Neurological cases associated with COVID-19 in pediatric patients have ranged from encephalitis to Guillain Barre and acute disseminated encephalomyelitis). ${ }^{3,6,17}$ There is one report of a 12-year-old patient with ischemic stroke who presented with seizures. ${ }^{18}$ In the series from Riphagen, one patient developed ischemic stroke while on extracorporeal membrane oxygenation (ECMO) support for shock. ${ }^{8}$ The unexpected number of cases that present with hemorrhagic stroke in Peru opens the possibility of describing a new presentation of COVID-19 in pediatric patients. It will be important to recollect data from countries with similar populations to identify risk factors and differentiate if it is a new phenotype or changes in the pathogenesis of the virus. Another important point is the need to have pediatric medical teams ready to manage this unusual presentation. Hemorrhagic and ischemic strokes in non-COVID pediatric patients have higher risks of morbidity and mortality if centers are not prepared with an appropriate neurocritical care infrastructure and team.

The neurological complications of COVID-19 have been described in detail in the adult literature. ${ }^{19-22}$ Studies examining patients who had stroke in adult populations described the event occurring in patients with severe presentation of SARS-CoV-2, and most of those patients had that event during their hospitalization. $^{21}$ In adults, the occurrence of this type of event has been described in different critical conditions, including viral infections. ${ }^{23}$ The physiopathology proposed for CVAs secondary to COVID-19 is the hyperinflammatory process, associated vasculitis, and coagulopathic states. ${ }^{22,24}$ It is unclear whether the same pathophysiology explains these events in pediatric patients. COVID-19 has been associated with inflammatory processes in pediatric patients, alterations of coagulation, and vasculitis, clearly seen in MIS-C. However, from the data presented in our study, the patients who had neurological manifestations had fewer comorbidities or preexisting conditions than the patients with respiratory failure and had similar or less severe inflammatory processes than the MIS-C patients, as noticed by the inflammatory markers. Additionally, not all the patients with neurological presentation in our cohort met the MIS-C criteria. Recognizing hemorrhagic and ischemic strokes as a presentation in pediatric patients with COVID19 is also especially important to make therapeutic decisions regarding anticoagulation in patients hospitalized with MIS-C.

As demonstrated by larger studies, comorbidities are associated with mortality in pediatric patients. ${ }^{1,3}$ The cohort we present shows this correlation. The comorbidities that our study presents are common diagnoses managed in pediatric critical care units, including congenital heart disease, leukemia, and cerebral palsy. An important detail about our study is that the group that had the most comorbidities was the acute COVID-19 group. All the patients who died from that group had a comorbidity and similar conditions to those described in a large series. ${ }^{1,3,12}$ Of the patients with neurological presentation, two patients had significant comorbidities, and both died during their admission. One of those conditions was undiagnosed leukemia that presented with hemorrhagic stroke and establishing causality with COVID-19 is difficult. The caveat to this is that there were five other patients with similar cerebrovascular events without comorbidities. The other patient with comorbidities from the neurological group was a patient with pulmonary tuberculosis who presented with meningitis and was thought to have COVID-19 on admission. Further studies to establish SARS-CoV-2 immunosuppression that would facilitate the pathogenesis of other infections are necessary. The government of Peru has approved ivermectin and 

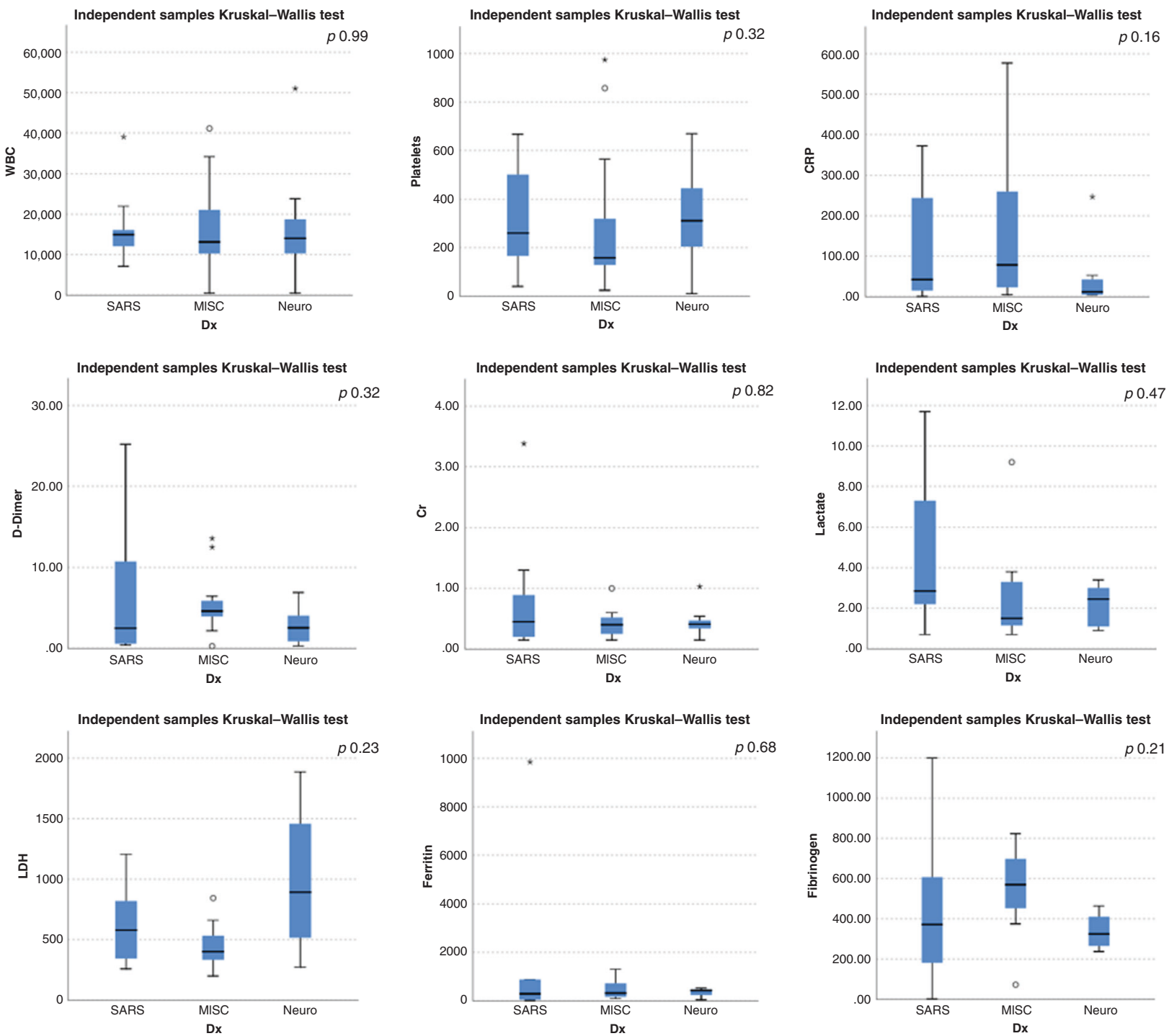

Fig. 2 Independent-Samples Kruskal-Wallis Test box plots for laboratory results. Diagnosis (Dx); white blood cell count (WBC) cells/mm3; platelets x 103; C-reactive protein (CRP) mg/L; D-dimer mcg/mL; creatinine (Cr) mg/dl; lactate mmol/L; lactate acid dehydrogenase (LDH) U/L; Ferritin $\mathrm{mcg} / \mathrm{L}$; fibrinogen $\mathrm{mg} / \mathrm{dl}$.

hydroxychloroquine as therapies approved for COVID-19. We did not find a difference in the utilization of those therapies across the three groups, and there was no correlation with mortality. Interestingly, steroids were more utilized in the acute and neurological groups than in the MIS-C group and did not correlate with mortality. There was no difference in vasoactive medication utilization or mortality outcomes. We did not find a difference in the laboratory results (peak value) in our patients. However, patients with higher WBC counts had higher mortality.

Since the management of pediatric critical care patients was centralized in one center, the patients included in our study were the sickest patients who were hospitalized in Peru, especially during the first two months of the pandemic. This setup had significant limitations: the patients who presented in emergency departments (EDs) that required critical care stayed in an observation area in that ED until a bed was available in the COVID-19 unit, and the transfer of the patient was possible. There is no clear information about the waiting time to have a bed available in the COVID-19 unit, which explains the high mortality of patients who were not able to be hospitalized in a PICU. By September 2020, according to the Peru Health Department (MINSA), 78 patients had died from COVID-19 in the group 0-9 years old, and 62 patients had died in the group 10-19 years old. A larger pediatric epidemiological study is necessary to understand the morbidity and mortality of patients who did not die in a pediatric critical care unit. We examined whether the mode of admission (local ED vs referring ED) and origin of reference (Lima vs other cities) had an impact on mortality. We did not find a difference between those variables. Having the waiting times could help establish if the model of a single center with six beds had an association with mortality. For a population of 10 million people younger than 18 years old, the number of beds designated for the PICU is likely not enough. The justification for a single center was to minimize the overutilization of protective equipment. The presentation of patients with strokes is challenging in any clinical setting, and it is difficult to argue that the outcomes we present would have been different in other countries.

Our study presents some limitations. Our number of patients is small compared to larger epidemiological studies. The acute presentation with neurological compromise and high mortality are higher than other studies in critical care. This study only captures the patients who were able to be hospitalized to the COVID-19 pediatric critical care unit and does not include a larger denominator. A larger national epidemiological study is necessary 
to determine if more cases of hemorrhagic strokes are present. The mortality of pediatric patients seems to be similar to that of patients in other countries, but the patients who were not included in our cohort died without accessing critical care. This is one of the challenges of the management of this pandemic in countries with developing health systems. Studies investigating viral characteristics in Peru are necessary.

\section{CONCLUSION}

Cerebrovascular events associated with COVID-19 in pediatric patients, including infants, must be recognized as one of the more severe presentations of this infection in pediatric patients. The management of these patients without critical care interventions can be fatal, and patients who survive can have severe sequelae. Recognizing this presentation in pediatric patients might help plan anticoagulation therapies for critical care patients with COVID-19.

\section{AUTHOR CONTRIBUTIONS}

All the authors participated in the conception, design, and acquisition of data. A.C.M. and J.D. analyzed and interpreted the data, and drafted the manuscript. All the authors approved the final version of the manuscript.

\section{ADDITIONAL INFORMATION}

Supplementary information The online version contains supplementary material available at https://doi.org/10.1038/s41390-021-01547-x.

Competing interests: The authors declare no competing interests.

Patient consent: No patient consent was required and was not obtained.

Publisher's note Springer Nature remains neutral with regard to jurisdictional claims in published maps and institutional affiliations.

\section{REFERENCES}

1. Dong, Y. et al. Epidemiology of COVID-19 Among Children in China. Pediatrics 145, e20200702 (2020).

2. Parri, N., Lenge, M. \& Buonsenso, D. Children with Covid-19 in pediatric emergency departments in Italy. N. Engl. J. Med. 383, 187-190 (2020).

3. Swann, O. V. et al. Clinical characteristics of children and young people admitted to hospital with covid-19 in United Kingdom: prospective multicentre observational cohort study. Bmj 370, m3249 (2020).

4. Belhadjer, Z. et al. Acute heart failure in multisystem inflammatory syndrome in children in the context of global SARS-CoV-2 Pandemic. Circulation 142, 429-436 (2020).
5. C. D. C. Multisystem Inflammatory Syndrome in Children (MIS-C) associated with Coronavirus Disease 2019 (COVID-19), case definition for MIS-C. In HAN (ed), https://emergency.cdc.gov/han/2020/han00432.asp (2020).

6. Feldstein, L. R. et al. Multisystem inflammatory syndrome in U.S. children and adolescents. N. Engl. J. Med. 383, 334-346 (2020).

7. Panupattanapong, S. \& Brooks, E. B. New spectrum of COVID-19 manifestations in children: Kawasaki-like syndrome and hyperinflammatory response. Cleve Clin. J. Med. Epub ahead of print (2020).

8. Riphagen, S., Gomez, X., Gonzalez-Martinez, C., Wilkinson, N. \& Theocharis, P. Hyperinflammatory shock in children during COVID-19 pandemic. Lancet 395, 1607-1608 (2020).

9. Toubiana, J. et al. Kawasaki-like multisystem inflammatory syndrome in children during the covid-19 pandemic in Paris, France: prospective observational study. BMJ 369, m2094 (2020).

10. Grimaud, M. et al. Acute myocarditis and multisystem inflammatory emerging disease following SARS-CoV-2 infection in critically ill children. Ann. Intensive Care 10, 69 (2020).

11. Kaushik, S. et al. Multisystem inflammatory syndrome in children associated with severe acute respiratory syndrome coronavirus 2 infection (MIS-C): a multiinstitutional study from New York city. J. Pediatr. 224, 24-29 (2020).

12. Shekerdemian, L. S. et al. Characteristics and outcomes of children with Coronavirus Disease 2019 (COVID-19) infection admitted to US and Canadian Pediatric Intensive Care Units. JAMA Pediatr. Sep 1 174, 868-873 (2020).

13. Garcia-Salido, A. et al. Children in critical care due to severe acute respiratory syndrome coronavirus 2 infection: experience in a Spanish hospital. Pediatr. Crit. Care Med. 24, 666 (2020).

14. González-Dambrauskas, S. et al. Pediatric critical care and COVID19. Pediatrics. 146, e20201766 (2020).

15. Sachdeva, R. et al. The impact of coronavirus disease 2019 pandemic on U.S. and Canadian PICUs. Pediatr. Crit. Care Med. 21, e643-e650 (2020).

16. Force, A. D. T. et al. Acute respiratory distress syndrome: the Berlin Definition. JAMA 307, 2526-2533 (2012).

17. Parsons, T. et al. COVID-19-associated acute disseminated encephalomyelitis (ADEM). J. Neurol. 267, 2799-2802 (2020).

18. Mirzaee, S. M. M., Gonçalves, F. G., Mohammadifard, M., Tavakoli, S. M. \& Vossough, A. Focal cerebral arteriopathy in a COVID-19 pediatric patient. Radiology. 297, E274-E275 (2020).

19. Ellul, M. A. et al. Neurological associations of COVID-19. Lancet Neurol. Sep 19, 767-783 (2020).

20. de Havenon, A., Ney, J. P., Callaghan, B., Yaghi, S. \& Majersik, J. J. Excess neurological death in New York City after the emergence of COVID-19. J. Neurol. Epub ahead of print (2020).

21. Li, Y. et al. Acute cerebrovascular disease following COVID-19: a single center, retrospective, observational study. Stroke Vasc. Neurol. 5, 279-284 (2020).

22. Spence, J. D. et al. Mechanisms of stroke in COVID-19. Cerebrovasc. Dis. 9 , 451-458 (2020).

23. Merkler, A. E. et al. Risk of ischemic stroke in patients with coronavirus disease 2019 (COVID-19) vs patients with influenza. JAMA Neurol. 77, 1-7 (2020).

24. Nordvig, A. S. et al. Potential neurological manifestations of COVID-19. Neurol. Clin. Pract. 11, e135-e146 (2020). 